\title{
THE STRUCTURE OF MULTIVARIATE SUPERSPLINE SPACES OF HIGH DEGREE
}

\author{
PETER ALFELD AND MARITZA SIRVENT
}

\begin{abstract}
We consider splines (of global smoothness $r$, polynomial degree $d$, in a general number $k$ of independent variables, defined on a $k$-dimensional triangulation $\mathscr{T}$ of a suitable domain $\Omega$ ) which are $r 2^{k-m-1}$-times differentiable across every $m$-face $(m=0, \cdots, k-1)$ of a simplex in $\mathscr{T}$. For the case $d>r 2^{k}$ we identify a structure that allows the construction of a minimally supported basis.
\end{abstract}

\section{INTRODUCTION}

A $\kappa$-simplex $K(0 \leq \kappa \leq k)$ is the convex hull of $\kappa+1$ points in $\mathbb{R}^{k}$ called the vertices of $K . K$ is nondegenerate if its $\kappa$-dimensional volume is nonzero, and degenerate otherwise. The dimension of a nondegenerate $\kappa$-simplex is $\kappa$. The convex hull of a subset of $\mu+1$ vertices of $K$ is a $\mu$-face of $K$.

Let $\mathscr{V} \subset \mathbb{R}^{k}$ be a given set of $N$ distinct points.

A triangulation $\mathscr{T}$ of the set $\mathscr{V}$ is a set of nondegenerate $k$-simplices satisfying the following requirements:

1. All vertices of each simplex in $\mathscr{T}$ are elements of $\mathscr{V}$.

2. The interiors of the simplices in $\mathscr{T}$ are pairwise disjoint.

3. The set

$$
\Omega:=\bigcup_{K \in \mathscr{J}} K \subset \mathbb{R}^{k}
$$

is homeomorphic to $[0,1]^{k}$.

4. Each $(k-1)$-face of a simplex in $\mathscr{T}$ is either on the boundary of $\Omega$, or else is a common face of exactly two simplices in $\mathscr{T}$.

5. No simplex in $\mathscr{T}$ contains any points of $\mathscr{V}$ other than its vertices.

Note that a $\mu$-face of a simplex in $\mathscr{T}$ is itself a $\mu$-dimensional simplex. On the triangulation $\mathscr{T}$ we define a spline space $S_{d}^{r}(\mathscr{T})$ as usual by

$$
S_{d}^{r}(\mathscr{T})=\left\{s \in C^{r}(\Omega):\left.s\right|_{\tau} \in \mathscr{P}_{d}^{k} \forall \tau \in \mathscr{T}\right\},
$$

where $\mathscr{P}_{d}^{k}$ is the $\left(\begin{array}{c}k+d \\ d\end{array}\right)$-dimensional linear space of all $k$-variate polynomials of total degree less than or equal to $d$.

Received February 27, 1990; revised September 21, 1990.

1980 Mathematics Subject Classification (1985 Revision). Primary 65D07, 41A63, 41A15. 
In this paper, we consider subspaces of $S_{d}^{r}(\mathscr{T})$ obtained by increasing the smoothness requirements across faces of the underlying simplices. More precisely, denoting by $\mathscr{S}_{\mu}$ the set of all $\mu$-faces of the simplices in $\mathscr{T}(\mu=$ $0, \ldots, k-1)$ and letting $\mathscr{S}=\bigcup_{\mu=0}^{k-1} \mathscr{S}_{\mu}$, we define the (superspline) space $\mathbb{S}_{d}^{r}(\mathscr{T})$ as a subspace of $S_{d}^{r}(\mathscr{T})$ as follows:

$$
\begin{array}{r}
\mathbb{S}_{d}^{r}(\mathscr{T})=\left\{s \in S_{d}^{r}(\mathscr{T}): s \text { is } \rho \text {-times differentiable across } \sigma \quad \forall \sigma \in \mathscr{S}\right\}, \\
\text { where } \rho=r 2^{k-\operatorname{dim} \sigma-1} .
\end{array}
$$

The concept of supersplines was introduced in Chui and Lai [8], [9]. The area in between finite elements and full spline spaces was further explored by Schumaker [16] and Ibrahim and Schumaker [11].

\section{THE GENERALIZED BEZIER-BERNSTEIN FORM}

Crucial to analyzing the dimension of spline spaces is the Bézier-Bernstein form of a multivariate polynomial. In the case $k \leq 2$ this form is used widely and is well known. A review of the Bézier-Bernstein form for a general number of variables is in de Boor [6]. In this paper, we use a notation that is particularly suitable for our purposes. However, generalized barycentric coordinates and global control nets have also been proposed in Alfeld [2] and de Boor [6].

We use $\mathscr{V}$ as an index set and denote by $\mathbb{N}$ the set of nonnegative integers. For vectors $\mathbf{I}=\left[i_{v}\right]_{v \in \mathscr{V}} \in \mathbb{N}^{N}$ and $\mathbf{a}=\left[a_{v}\right]_{v \in \mathscr{V}} \in \mathbb{R}^{N}$ we define

$$
\begin{aligned}
& |\mathbf{I}|=\sum_{v \in \mathscr{V}} i_{v}, \\
& \mathbf{a}^{\mathbf{I}}=|\mathbf{I}| ! \prod_{v \in \mathscr{V}} a_{v}^{i_{v}} / \prod_{v \in \mathscr{V}} i_{v} !,
\end{aligned}
$$

where

$$
0^{0}:=1
$$

We also use the notation

$$
\sigma(\mathbf{I})=\operatorname{conv}\left\{v: i_{v}>0\right\}, \quad \sigma(\mathbf{a})=\operatorname{conv}\left\{v: a_{v} \neq 0\right\}
$$

where conv $X$ denotes the convex hull of a point set $X$.

We now define generalized barycentric coordinates as cardinal piecewise linear functions $b_{v} \in S_{1}^{0}(\Omega)$ by the requirement

$$
b_{v}(w)=\delta_{v w}=\left\{\begin{array}{ll}
1 & \text { if } v=w \\
0 & \text { else }
\end{array} \forall v, w \in \mathscr{V} .\right.
$$

Clearly, in each $k$-simplex $K \in \mathscr{T}$ the functions $b_{v}$, where $v$ is a vertex of $K$, reduce to the ordinary barycentric coordinates. Globally, i.e., for all $x \in \Omega$, they satisfy

$$
\sum_{v \in \mathscr{V}} b_{v}=1, \quad b_{v} \geq 0 \quad \forall v \in \mathscr{V}, \quad \text { and } \quad x=\sum_{v \in \mathscr{V}} b_{v}(x) v .
$$


For a given polynomial degree $d$, we use the domain index set

$$
\mathbf{I}_{d}=\left\{\mathbf{I} \in \mathbb{N}^{N}:|\mathbf{I}|=d \text { and } \sigma(\mathbf{I}) \in \mathscr{S} \cup \mathscr{T}\right\} .
$$

Letting

$$
\mathbf{b}=\mathbf{b}(x)=\left[b_{v}(x)\right]_{v \in \mathscr{V}},
$$

it is clear that every function $s \in S_{d}^{0}(\mathscr{T})$ can be written as

$$
s=\sum_{\mathbf{I} \in \mathbf{I}_{d}} c_{\mathbf{I}} \mathbf{b}^{\mathbf{I}} .
$$

The coefficients $c_{\mathbf{I}}$ are the Bézier ordinates of $s$.

\section{The de Casteljau algorithm}

Let $s \in S_{d}^{0}(\mathscr{T}), K \in \mathscr{T}$, and $\left.s\right|_{K}=p \in \mathscr{P}_{d}^{k}$. Without loss of generality we may relabel the vertices and assume that $K$ is the $k$-simplex

$$
K=\left\{\mathbf{a}=\left(a_{1}, \cdots, a_{k+1}\right): a_{1}+\cdots+a_{k+1}=d, a_{j} \geq 0, a_{j} \in \mathbb{R}\right\} .
$$

Then, with $V_{j}$ denoting the vertices of $K$, we have

$$
p=\sum_{\mathbf{I} \in K \cap \mathbf{I}_{d}} c_{\mathbf{I}} \mathbf{b}^{\mathbf{I}} \quad \text { where } \quad x=\sum_{j=1}^{k+1} b_{j} V_{j}, \quad \sum_{j=1}^{k+1} b_{j}=1 .
$$

The Bernstein polynomials $\mathbf{b}^{\mathbf{I}}(x)$ satisfy a recurrence relation, see Farin [10]

$$
\mathbf{b}^{\mathbf{I}}(x)=\sum_{j=1}^{k+1} b_{j} \mathbf{b}^{\mathbf{I}-\varepsilon^{j}}(x), \quad|\mathbf{I}|=d, \quad \text { and } \quad \varepsilon^{j}=(0, \cdots, 1, \cdots, 0) .
$$

This relation allows one to expand $p$ in terms of Bernstein polynomials of lower degree with (polynomial) coefficients $p_{\mathbf{I}}^{r}(\mathbf{b})$.

Theorem 1. We have

$$
p=\sum_{|\mathbf{I}|=d-r} p_{\mathbf{I}}^{r}(\mathbf{b}) \mathbf{b}^{\mathbf{I}}, \quad 0 \leq r \leq d,
$$

where

$$
\begin{gathered}
p_{\mathbf{I}}^{0}(\mathbf{b})=c_{\mathbf{I}}, \\
p_{\mathbf{I}}^{r}(\mathbf{b})=\sum_{j=1}^{k+1} b_{j} p_{\mathbf{I}+\varepsilon^{j}}^{r-1}(\mathbf{b}), \quad|\mathbf{I}|=d-r, \quad 0 \leq r \leq d .
\end{gathered}
$$

The intermediate coefficients $p_{\mathbf{I}}^{r}(\mathbf{b})$ can also be written explicitly as

$$
p_{\mathbf{I}}^{r}(\mathbf{b})=\sum_{|\mathbf{M}|=r} c_{\mathbf{I}+M} \mathbf{b}^{\mathbf{M}}, \quad|\mathbf{I}|=d-r .
$$


The formulas in Theorem 1 may be used to evaluate $p$ at a given point, and are referred to as the de Casteljau Algorithm.

\section{SMOOTHNESS ACROSS AN INTERFACE}

Given a vector $e \in \mathbb{R}^{k}$, the directional derivative of $p$ in the direction of $\alpha=\left(\frac{\partial b_{1}}{\partial e}, \ldots, \frac{\partial b_{k+1}}{\partial e}\right)$, denoted $D_{\alpha}$, is given by (Alfeld [2])

$$
D_{\alpha} p=d \sum_{|\mathbf{I}|=d-1} p_{\mathbf{I}}^{1}(\alpha) \mathbf{b}^{\mathbf{I}}
$$

where

$$
p_{\mathbf{I}}^{1}(\alpha)=\sum_{|\mathbf{M}|=1} c_{\mathbf{I}+M} \boldsymbol{\alpha}^{\mathbf{M}}, \quad|\mathbf{I}|=d-1 .
$$

In general, the $r$ th directional derivative of $p$ in the direction of $\alpha$ is given by (Farin [10])

$$
D_{\alpha}^{r} p=\frac{d !}{(d-r) !} \sum_{|\mathbf{I}|=d-r} p_{\mathbf{I}}^{r}(\alpha) \mathbf{b}^{\mathbf{I}}
$$

where

$$
p_{\mathbf{I}}^{r}(\alpha)=\sum_{|\mathbf{M}|=r} c_{\mathbf{I}+M} \boldsymbol{\alpha}^{\mathbf{M}}, \quad|\mathbf{I}|=d-r, \quad \text { and } \quad p_{\mathbf{I}}^{0}(\alpha)=c_{\mathbf{I}} .
$$

The following theorem was proved by Farin [10] in the bivariate case; here we state without proof the result in any number of variables. Let $\tau$ be an $m$-face of $K$; without loss of generality, we will assume that

$$
\tau=\left\{\mathbf{a} \in K: a_{m+2}=\cdots=a_{k+1}=0\right\} .
$$

Theorem 2. Let $p, q \in \mathscr{P}_{d}^{k}$ be such that $\left.p\right|_{\tau}=\left.q\right|_{\tau}$. Then $D_{\alpha}^{s} p=D_{\alpha}^{s} q$ on $\tau$ for all directions $\alpha, 0 \leq s \leq r$, if and only if $p_{\mathbf{J}}^{r}=q_{\mathbf{J}}^{r}$ for all $\mathbf{J}=\left(j_{1}, \ldots, j_{m+1}\right.$, $0, \ldots, 0),|\mathbf{J}|=d-r$.

\section{SUBSIMPLICES AND SUBPOLYNOMIALS}

Let $K \in \mathscr{T}$ be a $k$-simplex and $\tau$ be a face of $K$ with $\operatorname{dim} \tau=n$,

$$
\begin{gathered}
K=\left\{\mathbf{a} \in \mathbb{R}^{N}: \sum_{v \in K} a_{v}=d, a_{v} \geq 0\right\}, \\
\tau=\left\{\mathbf{a} \in K: \sum_{v \in \tau} a_{v}=d\right\} .
\end{gathered}
$$

For $\mathbf{J} \in K \cap \mathbf{I}_{d}$, so that $\sum_{v \in \tau} j_{v}=d-\rho$, define

$$
\tau_{\mathbf{J}}=\left\{\mathbf{a}=\left[a_{v}\right]_{v \in K}: j_{v} \geq a_{v}, v \in \tau\right\} .
$$

Clearly, $\tau_{\mathbf{J}}$ is a subsimplex of $K$ similar to $K$. 
Let $p \in \mathscr{P}_{d}^{k}$, and $\left(\mathbf{I}, c_{\mathbf{I}}\right)_{\mathbf{I} \in K}$ be the control points of $p$ with respect to $K$. The control points $\left(\mathbf{I}, c_{\mathbf{I}}\right)_{\mathbf{I} \in \tau_{\mathrm{J}}}$ define a polynomial $p_{\mathbf{J}}$ of degree $\rho$. We call $\tau_{\mathbf{J}}$ the subsimplex of $K$ associated with $\mathbf{J}$, and $p_{\mathbf{J}}$ the subpolynomial of $p$ associated with $\mathbf{J}$.

De Boor [6] introduces the concepts of subsimplices and subpolynomials and proves most of the results of this section. Since our definitions are slightly different, we restate two key facts involving subpolynomials. Theorem 2 can be restated as follows:

Theorem 3. Let $p, q \in \mathscr{P}_{d}^{k}$, and $\tau$ be a face of $K$ with $\operatorname{dim} \tau=m$ such that $\left.p\right|_{\tau}=\left.q\right|_{\tau}$. Then

(28) $\quad D_{\alpha}^{s} p=D_{\alpha}^{s} q$ on $\tau$, for all directions $\alpha$ and $0 \leq s \leq \rho$,

if and only if

$$
p_{\mathbf{I}}=q_{\mathbf{I}} \quad \forall \mathbf{I} \in K \text { with } \operatorname{dist}(\mathbf{I}, \tau)=\rho .
$$

The above theorem immediately yields the following:

Theorem 4. Let $K, K^{\prime} \in \mathscr{T}, \tau \subset K \cap K^{\prime}, p, q \in \mathscr{P}_{d}^{k}$, and let $s \in S_{d}^{0}(\Omega)$ with $\left.s\right|_{K}=p,\left.s\right|_{K^{\prime}}=q$. Then $s \in C^{\rho}(\tau)$ if and only if $p_{\mathbf{I}}=q_{\mathbf{I}} \quad \forall \mathbf{I} \in K^{\prime}$ with $\operatorname{dist}(\mathbf{I}, \tau)=\rho$.

\section{DeTERMINING SETS}

We now generalize the concept of a determining set known from the bivariate case (see Alfeld and Schumaker [4]).

Definition 5. A set $D \subset \mathbf{I}_{d}$ is a determining set of $\mathbb{S}_{d}^{r}(\mathscr{T})$ if, for all $s \in \mathbb{S}_{d}^{r}(\mathscr{T})$,

$$
c_{\mathbf{I}}=0 \quad \forall \mathbf{I} \in D \Longrightarrow s \equiv 0 \text {. }
$$

$D$ is a minimal determining set if there is no determining set which has fewer elements than $D$.

It is clear from elementary linear algebra that the number of elements in a determining set of $\mathbb{S}_{d}^{r}(\mathscr{T})$ provides an upper bound on the dimension of $\mathbb{S}_{d}^{r}(\mathscr{T})$, and that the number of elements in a minimal determining set is unique and equals the dimension of $\mathbb{S}_{d}^{r}(\mathscr{T})$.

\section{A MiNiMAL DETERMINING SET OF $\mathbb{S}_{d}^{r}(\mathscr{T})$}

For each simplex $\sigma \in \mathscr{S} \cup \mathscr{T}$ let $\rho=r 2^{k-\operatorname{dim} \sigma-1}$ as before; we define two sets of domain indices recursively by

$$
\overline{\mathscr{D}}(\sigma)=\left\{\mathbf{I} \in \mathbf{I}_{d}: \sum_{v \in \sigma} i_{v} \geq d-\rho\right\}
$$

and

$$
\mathscr{D}(\sigma)=\overline{\mathscr{D}}(\sigma) \backslash \bigcup_{\tau \prec \sigma} \mathscr{D}(\tau)
$$

where $\tau$ is a proper face of $\sigma$. 
Definition 6. Let $\sigma \in \mathscr{S} \cup \mathscr{T}$. A set $\mathscr{A}(\sigma) \subset D(\sigma)$ is a determining set of $\overline{\mathscr{D}}(\sigma)$ if, for all $s \in \mathbb{S}_{d}^{r}(\mathscr{T})$,

$$
c_{\mathbf{I}}=0 \quad \forall \mathbf{I} \in \mathscr{A}(\sigma) \cup(\overline{\mathscr{D}}(\sigma) \backslash \mathscr{D}(\sigma)) \Longrightarrow c_{\mathbf{I}}=0 \quad \forall \mathbf{I} \in \overline{\mathscr{D}}(\sigma) .
$$

The set $\mathscr{A}$ is a minimal determining set of $\overline{\mathscr{D}}(\sigma)$ if there is no determining set of $\overline{\mathscr{D}}(\sigma)$ with fewer elements.

Lemma 7. Let $d>r 2^{k}$. Then, for all $\mathbf{I} \in I_{d}$ there exists a unique $\sigma \in \mathscr{S} \cup \mathscr{T}$ such that $\mathbf{I} \in \mathscr{D}(\sigma)$.

Proof. To prove the lemma, we have to show first that for all $\sigma, \tau \in \mathscr{S} \cup \mathscr{T}$ :

$$
\sigma \neq \tau \Longrightarrow \mathscr{D}(\sigma) \cap \mathscr{D}(\tau)=\varnothing \text {. }
$$

To establish this, suppose there is a domain index $I \in \mathscr{D}(\sigma) \cap \mathscr{D}(\tau)$, for two simplices $\sigma, \tau \in \mathscr{S} \cup \mathscr{T}$, such that $\operatorname{dim} \sigma \geq \operatorname{dim} \tau$, and $\tau$ is not a face of $\sigma$. Thus,

$$
\sum_{v \in \sigma} i_{v} \geq d-r 2^{k-\operatorname{dim} \sigma-1} \text { and } \sum_{v \in \tau} i_{v} \geq d-r 2^{k-\operatorname{dim} \tau-1},
$$

which implies

$$
\sum_{v \in \sigma} i_{v}+\sum_{v \in \tau} i_{v} \geq 2 d-r 2^{k-\operatorname{dim} \sigma-1}-r 2^{k-\operatorname{dim} \tau-1} .
$$

Moreover,

$$
\sum_{v \in \sigma} i_{v}+\sum_{v \in \tau} i_{v}=\sum_{v \in \sigma \cap \tau} i_{v}+\sum_{v \in \sigma \cup \tau} i_{v} .
$$

Now, rearranging (37), substituting (36) into (37), and using $\sum_{v \in \sigma \cup \tau} i_{v} \leq d$, we obtain

$$
\sum_{v \in \sigma \cap \tau} i_{v} \geq d-r 2^{k-\operatorname{dim} \tau}
$$

So there exists a face $\tilde{\tau}$ of $\tau$ such that $\mathbf{I} \in \mathscr{D}(\tilde{\tau})$, which is a contradiction.

Finally, we need to prove that $\mathbf{I}_{d}=\bigcup_{\sigma \in \mathscr{S} \cup \mathscr{G}} \mathscr{D}(\sigma)$.

To see this, we only need to show that for each domain index $\mathbf{I} \in \mathbf{I}_{d}$ there exists a simplex $\sigma \in \mathscr{S} \cup \mathscr{T}$ such that $\mathbf{I} \in \mathscr{D}(\sigma)$. However, this is trivial, since for each domain index $I$ there exists at least one $k$-simplex $K$ such that $\sum_{v \in K} i_{v}=d$. Thus, $\mathbf{I} \in \overline{\mathscr{D}}(K)$, and there must be a simplex $\sigma \prec K$ such that $\mathbf{I} \in \mathscr{D}(\sigma)$.

Lemma 8. Let $d>r 2^{k}, \rho=r 2^{k-\operatorname{dim} \sigma-1}$, and $\mathscr{A}(\sigma):=\mathscr{D}(\sigma) \cap K$, where $K \in \mathscr{T}$ and $\sigma \prec K$. Then $\mathscr{A}(\sigma)$ is a minimal determining set of $\overline{\mathscr{D}}(\sigma)$.

Proof. First we establish that $\mathscr{A}(\sigma)$ is determining.

Let $s \in \mathbb{S}_{d}^{r}(\mathscr{T})$, and let $\left.s\right|_{K}=p$, with $p \in \mathscr{P}_{d}^{k}$. If

$$
c_{\mathbf{I}}=0 \quad \forall \mathbf{I} \in \mathscr{A}(\sigma) \cup(\overline{\mathscr{D}}(\sigma) \backslash \mathscr{D}(\sigma)),
$$

then

$$
p_{\mathbf{I}}=0 \quad \forall \mathbf{I} \in \mathscr{A}(\sigma) \cup(\overline{\mathscr{D}}(\sigma) \backslash \mathscr{D}(\sigma)),
$$


where $p_{\mathrm{I}}$ is the subpolynomial of $p$ of degree $\leq \rho$ associated with I (notice that dist $(\mathbf{I}, \sigma) \leq \rho \quad \forall \mathbf{I} \in \mathscr{A}(\sigma))$. Let $K^{\prime}$ be any other $k$-simplex of $\mathscr{T}$ such that $\sigma \prec K^{\prime}$ and $K \neq K^{\prime}$, and let $q=\left.s\right|_{K^{\prime}}, q \in \mathscr{P}_{d}^{k}$. Now, $s \in C^{\rho}(\sigma)$; then $\forall \mathbf{I} \in\left(\mathscr{D}(\sigma) \cap K^{\prime}\right) \cup(\overline{\mathscr{D}}(\sigma) \backslash \mathscr{D}(\sigma)) p_{\mathbf{I}}=q_{\mathbf{I}}$, where $q_{\mathbf{I}}$ is the subpolynomial of $q$ of degree $\leq \rho$ associated with $I$. But,

$$
\begin{gathered}
p_{\mathbf{I}}=0 \quad \forall \mathbf{I} \in(\mathscr{D}(\sigma) \cap K) \cup(\overline{\mathscr{D}}(\sigma) \backslash \mathscr{D}(\sigma)) \\
\Longrightarrow \quad q_{\mathbf{I}}=0 \quad \forall \mathbf{I} \in\left(\mathscr{D}(\sigma) \cap K^{\prime}\right) \cup(\overline{\mathscr{D}}(\sigma) \backslash \mathscr{D}(\sigma)) \\
\Longrightarrow \quad c_{\mathbf{I}}=0 \quad \forall \mathbf{I} \in\left(\mathscr{D}(\sigma) \cap K^{\prime}\right) \cup(\overline{\mathscr{D}}(\sigma) \backslash \mathscr{D}(\sigma)) \\
\Longrightarrow \quad c_{\mathbf{I}}=0 \quad \forall \mathbf{I} \in\left(\bigcup_{\substack{K \in \mathscr{F} \\
\sigma \prec K}} \mathscr{D}(\sigma) \cap K\right) \cup(\overline{\mathscr{D}}(\sigma) \backslash \mathscr{D}(\sigma)) \\
\Longrightarrow \\
c_{\mathbf{I}}=0 \quad \forall \mathbf{I} \in \mathscr{D}(\sigma) \cup(\overline{\mathscr{D}}(\sigma) \backslash \mathscr{D}(\sigma)) .
\end{gathered}
$$

Therefore,

$$
c_{\mathbf{I}}=0 \quad \forall \mathbf{I} \in \overline{\mathscr{D}}(\sigma) .
$$

To see that $\mathscr{A}(\sigma)$ is indeed minimal, take $\mathbf{I} \in \mathscr{A}(\sigma)$ and consider the set $\widetilde{\mathscr{A}}(\sigma)=\mathscr{A}(\sigma) \backslash\{\mathbf{I}\}$, define a polynomial on $K$ whose Bézier coefficients are equal to zero except at the domain point $\mathbf{I}$, where $c_{I}=1$, and extend this polynomial globally on the rest of the triangulation.

The coefficients $c_{\mathrm{J}}$ are equal to zero on $\mathscr{D}(\sigma) \backslash \mathscr{D}(\sigma)$, since the smoothness conditions there only involve domain indices in $\mathscr{D}(\tau)$ for $\tau \prec \sigma$.

Hence, $c_{\mathbf{J}}=0 \quad \forall J \in \widetilde{\mathscr{A}}(\sigma) \cup(\overline{\mathscr{D}}(\sigma) \backslash \mathscr{D}(\sigma))$, but this does not imply that $c_{\mathbf{J}}=0 \quad \forall J \in \overline{\mathscr{D}}(\sigma)$ since in particular $I \in \overline{\mathscr{D}}(\sigma)$ and $c_{\mathbf{I}}=1$.

Therefore, $\widetilde{\mathscr{A}}(\sigma)$ cannot be determining and $\mathscr{A}(\sigma)$ is a minimal determining set of $\overline{\mathscr{D}}(\sigma)$.

The following theorem is the central result of this paper.

Theorem 9. Let $r \geq 0, d>r 2^{k}$ and let $\mathscr{A}(\sigma)=\mathscr{D}(\sigma) \cap K$, where $K \in \mathscr{T}$ is a $k$-simplex so that $\sigma$ is a face of $K$. Then

$$
\mathscr{A}:=\bigcup_{\sigma \in \mathscr{S} \cup T} \mathscr{A}(\sigma)
$$

is a minimal determining set of $\mathbb{S}_{d}^{r}(\mathscr{T})$.

Proof. Let $s \in \mathbb{S}_{d}^{r}(\mathscr{T})$ and assume that $c_{\mathbf{I}}=0 \quad \forall I \in \mathscr{A}$. Using induction on $\operatorname{dim} \sigma$, we first establish that $\mathscr{A}$ is a determining set.

(1) If $\operatorname{dim} \sigma=0$ then $c_{\mathbf{I}}=0 \quad \forall I \in \mathscr{A}(\sigma)$ implies $c_{\mathbf{I}}=0 \quad \forall I \in \overline{\mathscr{D}}(\sigma)$, since $\mathscr{D}(\sigma)=\overline{\mathscr{D}}(\sigma)$.

(2) We assume now that $c_{\mathrm{I}}=0 \quad \forall I \in \overline{\mathscr{D}}(\sigma)$ and $\forall \sigma$ with $\operatorname{dim} \sigma<n$. 
(3) Let $\operatorname{dim} \sigma=n, c_{\mathrm{I}}=0 \quad \forall I \in \mathscr{A}(\sigma)$. Let $\mathscr{B}(\sigma)=\mathscr{A}(\sigma) \cup(\overline{\mathscr{D}}(\sigma) \backslash \mathscr{D}(\sigma))$; notice that $B(\sigma) \subset \mathscr{A}(\sigma) \cup\left(\bigcup_{\tau \prec \sigma} \overline{\mathscr{D}}(\tau)\right)$.

By the induction hypothesis, $c_{\mathrm{I}}=0 \quad \forall I \in \bigcup_{\tau \prec \sigma} \overline{\mathscr{D}}(\tau)$ implies that $c_{\mathrm{I}}=0$ $\forall I \in \mathscr{B}(\sigma)$. But $\mathscr{A}(\sigma)$ is a determining set of $\overline{\mathscr{D}}(\sigma)$; thus, $c_{\mathrm{I}}=0 \forall I \in \overline{\mathscr{D}}(\sigma)$, hence

$$
c_{\mathrm{I}}=0 \quad \forall I \in \bigcup_{\sigma \in \mathscr{S} \cup T} \overline{\mathscr{D}}(\sigma),
$$

and by Lemma 7 we get that

$$
c_{\mathbf{I}}=0 \quad \forall \mathbf{I} \in \mathbf{I}_{d} .
$$

To show that $\mathscr{A}$ is indeed minimal, we give arbitrary Bézier ordinates $c_{I}$ $\forall \mathbf{I} \in \mathscr{A}$, and we construct the rest of the Bézier ordinates in such a way that the piecewise polynomial they determine is a superspline.

So, we assume $c_{\mathbf{I}} \forall \mathbf{I} \in \mathscr{A}$ are given, and let $\mathbf{J} \in \mathbf{I}_{d}$. Then by Lemma 7 there is a unique $\sigma \in \mathscr{S} \cup T$ such that $\mathbf{J} \in \mathscr{D}(\sigma)$.

We define $c_{\mathbf{J}}$ inductively over $\operatorname{dim} \sigma$.

(1) If $\sigma$ is a vertex, then there exists $K \in \mathscr{T}$ so that $\sigma \prec K$ and $\mathscr{A}(\sigma) \subset K$.

Let $\sigma_{\mathbf{J}}$ be the subsimplex of $K$ associated with $\mathbf{J}, \sigma_{\mathbf{J}} \subset \mathscr{A}(\sigma)$. Then the polynomial $p_{\mathrm{J}}$ is defined; so, for $\mathrm{J} \in \mathscr{D}(\sigma) \cap K^{\prime}$ with $K^{\prime} \in \mathscr{T}, K^{\prime} \neq K$, let $\tau_{\mathbf{J}}$ be the subsimplex of $K^{\prime}$ associated with $\mathbf{J}$. Then there is a unique way to give Bézier ordinates to the domain points in $\tau_{\mathbf{J}}$ in such a way that they define a polynomial $q_{\mathbf{J}}$ which equals $p_{\mathbf{J}}$. Furthermore, in this manner, $c_{\mathbf{J}}$ can be defined for all $\mathbf{J} \in \mathscr{D}(\sigma) \backslash \mathscr{A}(\sigma)$.

(2) Suppose $c_{\mathbf{J}}$ has been defined for all $\mathbf{J} \in \bigcup_{\operatorname{dim} \sigma<n} \mathscr{D}(\sigma)$.

(3) Let $\mathbf{J} \in \mathscr{D}(\sigma)$ with $\sigma$ an $n$-simplex and $K \in \mathscr{T}$ such that $\mathscr{A}(\sigma) \subset K$. And let again $\sigma_{\mathbf{J}}$ be the subsimplex of $K$ associated with $\mathbf{J}$. Note that

$$
\sigma_{\mathbf{J}} \subset \overline{\mathscr{D}}(\sigma) \cap K \subset \mathscr{A}(\sigma) \cup\left(\bigcup_{\tau \prec \sigma} \mathscr{D}(\tau) \cap K\right) .
$$

Then by the induction hypothesis and by the fact that the Bézier ordinates have been defined on $\mathscr{A}$, we have that all of the Bézier ordinates on $\sigma_{\mathrm{J}}$ are defined; therefore $p_{\mathbf{J}}$ is defined. So as before, for $\mathbf{J} \in \mathscr{D}(\sigma) \cap K^{\prime}, K^{\prime} \neq K$, we can define $q_{\mathbf{J}}$ in the same way we did when $\sigma$ was a vertex. Thus, $c_{\mathrm{I}}$ has been defined for all $\mathbf{I} \in \mathbf{I}_{d}$.

Next, we need to show that the piecewise polynomial function defined by the $c_{\mathrm{I}}$ 's is well defined.

Suppose $p_{\mathbf{L}}=q_{\mathbf{L}}$ for $\mathbf{L} \in K$ and $\sum_{v \in \xi} l_{v}=d-\rho$.

Let $\mathbf{J} \in \mathscr{D}(\sigma) \cap K^{\prime}$ and suppose that $\mathbf{J} \in \xi_{\mathbf{L}}$ with $\mathbf{J} \neq \mathbf{L}$, where

$$
\xi_{\mathbf{L}}=\left\{\mathbf{I} \in K^{\prime}: i_{v} \geq l_{v} v \in \xi, \quad \xi \in \mathscr{S} \cup T, \text { and } \sigma \prec \xi\right\}
$$

and

$$
\tau_{\mathbf{J}}=\left\{\mathbf{I} \in K^{\prime}: i_{v} \geq j_{v} \quad v \in \sigma\right\}
$$


Then $\mathbf{J} \in \xi_{\mathbf{L}}$ implies $j_{v} \geq l_{v}$, which in turn implies $\tau_{\mathbf{J}} \subset \xi_{\mathbf{L}}$. Therefore, $q_{\mathbf{J}}$ is a subpolynomial of $q_{\mathrm{L}}$. Similarly, $p_{\mathrm{J}}$ is a subpolynomial of $p_{\mathrm{L}}$. Hence, $p_{\mathrm{J}}=q_{\mathrm{J}}$ since $q_{\mathrm{L}} \cup p_{\mathrm{L}} \in C^{\infty}(\xi)$. Therefore, the Bézier ordinates are well defined and by construction, the piecewise polynomial defined is a superspline.

Corollary 10. We have $\operatorname{dim} \mathbb{S}_{d}^{r}(\mathscr{T})=\sum_{\sigma \in \mathscr{S} \cup \mathscr{T}}|\mathscr{A}(\sigma)|$.

In view of the above corollary, to compute $\operatorname{dim} \mathbb{S}_{d}^{r}(\mathscr{T})$ we need only to know the cardinality of $\mathscr{A}(\sigma)$ for every $\sigma \in \mathscr{S}$. Clearly, $|\mathscr{A}(\sigma)|$ depends only on $m=\operatorname{dim} \sigma$. The following theorem is proved in Alfeld and Sirvent [5].

Theorem 11. We have $\operatorname{dim} \mathbb{S}_{d}^{r}(\mathscr{T})=\sum_{m=0}^{k} \phi(m) f_{m}$, where $f_{m}$ is the number of $m$-simplices of $\mathscr{S} \cup \mathscr{T}$, and for $m=0, \ldots, k, \phi(m)=\phi_{m}^{k}(0)$, where, with $\rho_{m}=r 2^{k-m-1}$, the quantities $\phi_{q}^{m}(p)$ are defined recursively by

$$
\begin{aligned}
\phi_{q}^{m}(p)=\sum_{j=0}^{\rho_{q}-p}\left(\begin{array}{c}
j+m-q-1 \\
j
\end{array}\right)[ & \left(\begin{array}{c}
d-p-j+q \\
q
\end{array}\right) \\
& \left.-\sum_{i=0}^{q-1}\left(\begin{array}{c}
q+1 \\
i+1
\end{array}\right) \phi_{i}^{q}(p+j)\right]
\end{aligned}
$$

if $0<q<m$, and

$$
\begin{gathered}
\phi_{0}^{m}(p)=\sum_{j=0}^{\rho_{0}-p}\left(\begin{array}{c}
j+m-1 \\
j
\end{array}\right)=\left(\begin{array}{c}
\rho_{0}-p+m \\
m
\end{array}\right), \\
\phi_{k}^{k}(0)=\left(\begin{array}{c}
d+k \\
k
\end{array}\right)-\sum_{m=0}^{k-1}\left(\begin{array}{l}
k+1 \\
m+1
\end{array}\right) \phi_{m}^{k}(0) .
\end{gathered}
$$

\section{Minimally SUPPORTED BASES}

Definition 12. The star of a simplex $\sigma \in \mathscr{S} \cup T$, denoted $\operatorname{star}(\sigma)$, is the set of all $k$-simplices $K \in \mathscr{T}$ such that $\sigma$ is a face of $\mathrm{K}$.

Definition 13. A basis $\left\{l_{\mu}: \mu=1,2, \ldots, \operatorname{dim} \mathbb{S}_{d}^{r}(\mathscr{T})\right\}$ is said to be minimally supported if for each basis function $l_{\mu}$ there exists a simplex $\sigma \in \mathscr{S} \cup T$ such that the support of $l_{\mu}$ is contained in $\operatorname{star}(\sigma)$.

The basis functions constructed in the proof of Theorem 9 are minimally supported: using the same construction, we can define cardinal supersplines

$$
l_{\mathbf{I}} \in \mathbb{S}_{d}^{r}(\mathscr{T}): \quad l_{\mathbf{I}}(\mathbf{J})=\delta_{\mathbf{I J}} \quad \forall \mathbf{I}, \mathbf{J} \in \mathscr{A}
$$

so that, if $\mathbf{I} \in \mathscr{A}(\sigma)$, then $c_{\mathbf{J}}=0 \quad \forall \mathbf{J} \in \mathbf{I}_{d} \backslash \operatorname{star}(\sigma)$ and $c_{\mathbf{I}}=1$.

\section{ACKNOWLEDGMENTS}

This research was supported by the National Science Foundation with grant DMS-8701121 to the University of Utah. The authors also appreciate the careful work of the editor and the thoughtful comments on an earlier version of this paper by an anonymous referee. 


\section{BIBLIOGRAPHY}

1. P. Alfeld, Scattered data interpolation in three or more variables, Mathematical Methods in Computer Aided Geometric Design (Tom Lyche and Larry L. Schumaker eds.), Academic Press, New York, 1989, pp. 1-34.

2. _ A bivariate $C^{2}$ Clough-Tocher scheme, Comput. Aided Geom. Design 1 (1984), 257267.

3. Peter Alfeld, B. Piper, and L. L. Schumaker, Minimally supported bases for spaces of bivariate piecewise polynomials of smoothness $r$ and degree $d \geq 4 r+1$, Comput. Aided Geom. Design 4 (1987), 105-123.

4. Peter Alfeld and L. L. Schumaker, The dimension of bivariate spline spaces of smoothness $r$ for degree $d \geq 4 r+1$, Constr. Approx. 3 (1987), 189-197.

5. Peter Alfeld and $\mathrm{M}$. Sirvent, $A$ recursion formula for the dimension of superspline spaces of smoothness $r$ and degree $d>r 2^{k}$, Approximation Theory $\mathrm{V}$ (W. Schempp and $\mathrm{K}$. Zeller, eds.), (Proceedings of the Oberwolfach Meeting, February 12-18, 1989), Birkhäuser Verlag, Basel, 1989, pp. 1-8.

6. C. de Boor, B-form basics, Geometric Modeling: Algorithms and New Trends (G. E. Farin, ed.), SIAM, Philadelphia, PA, 1987, pp. 131-148.

7. C. K. Chui, Multivariate splines, SIAM, Philadelphia, PA, 1988.

8. C. K. Chui and M. J. Lai, On multivariate vertex splines and applications, Topics in Multivariate Approximation (C. K. Chui, L. L. Schumaker, and F. Utreras, eds.), Academic Press, New York, 1987, pp. 19-36.

9. On bivariate vertex splines, Multivariate Approximation Theory III (W. Schempp and K. Zeller, eds.), Birkhäuser Verlag, Basel, 1985, pp. 84-115.

10. G. Farin, Triangular Bernstein-Bézier patches, Comput. Aided Geom. Design 3 (1986), 83-128.

11. A. Ibrahim and L. L. Schumaker, Superspline spaces of smoothness $r$ and degree $d \geq 3 r+2$, Constr. Approx., 1991.

12. C. L. Lawson, Properties of n-dimensional triangulations, Comput. Aided Geom. Design 3 (1986), 231-247.

13. J. Morgan and R. Scott, $A$ nodal basis for $C^{1}$ piecewise polynomials of degree $n \geq 5$, Math. Comp. 29 (1975), 736-740.

14. L. L. Schumaker, On the dimension of spaces of piecewise polynomials in two variables, Multivariate Approximation Theory (W. Schempp and K. Zeller, eds.), Birkhäuser Verlag, Basel, 1979, pp. 396-412.

15. _ Bounds on the dimension of spaces of multivariate piecewise polynomials, Rocky Mountain J. Math. 14 (1984), 251-264.

16. __ On super splines and finite elements, SIAM J. Numer. Anal. 26 (1989), 997-1005.

17. G. Strang, Piecewise polynomials and the finite element method, Bull. Amer. Math. Soc. 79 (1973), 1128-1137.

Department of Mathematics, University of Utah, Salt Lake City, Utah 84112

E-mail address: alfeld@math.utah.edu 